\title{
A REMARK ON A RESULT OF MCKEAN
}

\author{
MARYSIA TARNOPOLSKA-WEISS
}

\begin{abstract}
The diameter of the Dirichlet polygon associated to certain discontinuous groups acting on the upper half-plane is shown to be bounded. This clarifies a point in the proof of a result of McKean.
\end{abstract}

In a very useful paper [2] McKean proves the following interesting result: There are, up to isometry, only finitely many compact Riemann surfaces $M$ corresponding to a given spectrum of the Laplacian on $M$. Here we are regarding $M$ as the quotient of the upper half-plane $H^{+}$by a discontinuous group $\Gamma$ of hyperbolic transformations and assuming that $\mathrm{H}^{+}$is endowed with the metric $\left((d x)^{2}+(d y)^{2}\right) / y^{2}$.

There is a point in McKean's proof of this result which is not completely obvious, and it is the purpose of this note to give a simple way around this. The problem involves bounding the diameter of a fundamental polygon, $S_{\Gamma}$, for $\Gamma$ in terms of the diameter of $M$ itself. This can easily be circumvented by the following result.

Theorem. Let $S_{\Gamma}$ be the Dirichlet polygon for $\Gamma$ centered at i, i.e. the fundamental domain bounded by segments of perpendicular bisectors of the geodesics joining $i$ and its translates by $\Gamma$. Then, provided the genus of $M$ is fixed and there is a lower bound on the shortest closed geodesic (which is automatically furnished by the Selberg trace formula, when the spectrum is given), $\exists$ a constant $C>0$ such that diam $\left(S_{\Gamma}\right) \leqslant C$ for all $\Gamma$.

We will prove the Theorem by contradiction. Suppose $\left\{\Gamma_{r}\right\}$ is a sequence of discontinuous groups consisting of hyperbolic transformations, each having compact $S_{\Gamma_{r}}$ and such that diam $\left(S_{\Gamma_{r}}\right) \rightarrow \infty$, and for all $r$ the corresponding Riemann surfaces have the same fixed genus $g$, i.e. meas $\left(S_{\Gamma_{r}}\right)=A$, where $A=4 \pi(g-1)$. Assume also that for all $r$, $\min l_{\gamma_{r}}$, the length of the shortest closed geodesic, is greater than $\varepsilon$ for some $\varepsilon>0$.

Then if $\gamma_{r} \in \Gamma_{r}$ is not the identity, $\left|\operatorname{sp}\left(\gamma_{r}\right)\right| \geqslant 2+\eta(\varepsilon)$, where $\left|\operatorname{sp}\left(\gamma_{r}\right)\right|$ is the absolute value of the trace of $\gamma_{r}$ (this is well defined for $\gamma \in \operatorname{PSL}(2, R)$ ), and $\eta(\varepsilon)=2 \cosh \varepsilon / 2-2$. Let $V$ be the set of transformations such that if $\gamma \in V$, then $|\operatorname{sp}(\gamma)|<2+\eta(\varepsilon)$. Clearly $\Gamma_{r} \cap V=e$ for all $r(e$ is the identity element of $G=\operatorname{PSL}(2, R))$. Now our sequence of discontinuous groups satisfies the hypotheses of Theorem 1 and Lemma 7 of [1], which, adjusted to our case, state that: If $\left\{\Gamma_{r}\right\}$ is a sequence of lattices in $G$ and if (1) $\exists$ an open neighborhood $V$ of $e$ such that $\Gamma_{r} \cap V=e$ for all $r$, and (2) $\exists$ a constant $A<\infty$ such that meas $\left(G / \Gamma_{r}\right) \rightarrow A$, then one can extract from $\left\{\Gamma_{r}\right\}$ a

Received by the editors June 15, 1975.

AMS (MOS) subject classifications (1970). Primary 22E40. 
subsequence $\left\{\Gamma_{r^{\prime}}\right\}$ which converges to a lattice $\Gamma$ with $\Gamma \cap V=e$ and meas $(G / \Gamma)=A$. Here $\Gamma_{r^{\prime}} \rightarrow \Gamma$ means that if $U$ is any neighborhood of $e$ in $G$, and $K$ is a compact set in $G$, then for $r$ sufficiently large, to each $\gamma \in \Gamma_{r} \cap K$ there corresponds an $\alpha \in \Gamma$ such that $\alpha^{-1} \gamma \in U$, and for each $\alpha \in \Gamma \cap K$ there is $\gamma \in \Gamma_{r}$ such that $\alpha^{-1} \gamma \in U$. Thus our sequence $\left\{\Gamma_{r}\right\}$ has a subsequence $\left\{\Gamma_{r^{\prime}}\right\}$ converging in this sense to a discontinuous group $\Gamma$, with $\Gamma \cap V=e$, and meas $\left(S_{\Gamma}\right)=A$.

Claim. $S_{\Gamma}$ is compact. If not, $\Gamma$ must admit parabolic transformations and this cannot happen since, apart from the identity, all $\gamma \in \Gamma$ satisfy $|\operatorname{sp}(\gamma)|$ $\geqslant 2+\eta(\varepsilon)$. Thus the diameter of $S_{\Gamma}$ is bounded and if $\Gamma_{r} \rightarrow \Gamma$, diam $\left(S_{\Gamma_{r}}\right)$ $\rightarrow \operatorname{diam}\left(S_{\Gamma}\right)$. The last statement follows immediately from the definition of the limit of a sequence of discontinuous groups. Let $g_{1}, g_{2}, \ldots, g_{k}$ be the set of generators of $\Gamma$ which give the arcs of $S_{\Gamma}$. Then for $r$ large enough we can find $g_{1}^{r}, g_{2}^{r}, \ldots, g_{k}^{r}$ generators of $\Gamma_{r}$ which give the arcs of $S_{\Gamma_{r}}$ and $g_{i}^{r} \rightarrow g_{i}$, so we are done.

The bound on the diameter of a fundamental domain is used in McKean's paper to show that if $g_{1}, g_{2}, \ldots, g_{n}$ are generators of $\Gamma$, then $\left|\operatorname{sp}\left(g_{i}\right)\right|$, $\left|\mathrm{sp}\left(g_{i} g_{j}\right)\right|$, and $\left|\mathrm{sp}\left(g_{i} g_{j} g_{k}\right)\right|$ are bounded. This together with the fact that sp $\left(g_{i}\right)$, sp $\left(g_{i} g_{j}\right)$ and sp $\left(g_{i} g_{j} g_{k}\right)$ determine $\Gamma$ up to conjugation in PSL $(2, R)$ and/or reflection completes McKean's proof.

\section{REFERENCES}

1. C. Chabauty, Limite d'ensembles et géométrie des nombres, Bull. Soc. Math. France 78 (1950), 143-151. MR 12, 479.

2. H. P. McKean, Jr., Selberg's trace formula as applied to a compact Riemann surface, Comm. Pure Appl. Math. 25 (1972), 225-246.

Department of Mathematics, Queens College, City University of New York, Flushing, New YoRK 11367 